Check for updates

Cite this: Chem. Commun., 2017, 53,8455

Received 26th June 2017 Accepted 29th June 2017

DOI: $10.1039 / c 7 c c 04945 e$

rsc.li/chemcomm

\section{Low-toxicity metallosomes for biomedical applications by self-assembly of organometallic metallosurfactants and phospholipids $\dagger$}

\author{
M. Marín-García, (D) ${ }^{a}$ N. Benseny-Cases, (D) ${ }^{b}$ M. Camacho, (D) ${ }^{c}$ J. Suades (DD ${ }^{d}$ and \\ R. Barnadas-Rodríguez (D) *a
}

\begin{abstract}
A new and convenient strategy for the preparation of metallosomes has been developed by mixing organometallic metallosurfactants and phospholipids. These aggregates show the characteristic properties of liposomes (stability upon dilution and low toxicity) and the toxicity is at least ten-fold lower than that of the metallosurfactant aggregates without phospholipids.
\end{abstract}

The preparation and study of nanomaterials by self-assembly in aqueous medium is an increasingly relevant topic. Supramolecular structures for a wide range of applications can be obtained from simple molecules, which can be easily modified to modulate the properties of the aggregates in order to yield new materials with designed properties. Surfactants that contain a metal atom in the molecular structure are named metallosurfactants (MTSs) and they are attractive molecules due to the fact that they can lead to characteristic supramolecular aggregates (micelles, vesicles, etc.), and simultaneously they contain metallic atoms. The presence of the metal makes it possible to use these aggregates in a broad range of applications such as catalysis, optoelectronics, and biomedicine. ${ }^{1}$ Although in most of the reported MTS the metallic atom is located in the polar group of the molecule, we have recently reported organometallic MTSs wherein the metallic fragment is embedded in the hydrophobic part of the molecule. ${ }^{2}$ These MTSs render mostly unilamellar vesicles in water if the MTS concentration is higher than the critical value. However, dilution of these suspensions leads to disaggregation of the vesicles, which is a drawback of using them in in vivo applications. To circumvent

\footnotetext{
${ }^{a}$ Biophysics Unit/Center for Biophysical Studies, Department of Biochemistry and Molecular Biology, Faculty of Medicine, Universitat Autònoma de Barcelona, Avda. de Can Domènech, 08193 Cerdanyola, Spain.E-mail: Ramon.Barnadas@uab.cat

${ }^{b}$ ALBA Synchrotron, Carrer de la Llum 2-26, 08290 Cerdanyola, Spain

${ }^{c}$ Laboratory of Angiology, Vascular Biology and Inflammation/Institute of Biomedical Research, Hospital de la Santa Creu i de Sant Pau, Universitat Autònoma de Barcelona, Sant Antoni Maria Claret, 167, 08025 Barcelona, Spain

${ }^{d}$ Departament de Química, Edifici C, Universitat Autònoma de Barcelona, o8193 Cerdanyola, Spain.E-mail: Joan.Suades@uab.cat

$\dagger$ Electronic supplementary information (ESI) available: Materials and methods with experimental details, supporting Fig. S1-S4. See DOI: 10.1039/c7cc04945e
}

this disadvantage we considered the possibility of obtaining mixed systems with phospholipids. In particular, we used soybean phosphatidylcholine (SPC), a natural non-toxic phospholipid that is known to form the so-called liposomes, that is, dilution-stable closed vesicles with entrapped water (Scheme 1), and which are used for several medical applications. ${ }^{3}$ Our hypothesis is that mixed systems of MTS and phospholipids (Scheme 1) could lead to new supramolecular aggregates with a higher biocompatibility and with useful properties for biomedical applications. Since these new systems contain metallic atoms in the liposome membrane, they can be classified as a new kind of metallosome. ${ }^{4}$ In this article, we report the study of the viability of the preparation of metallosomes by mixing SPC with two organometallic MTSs, the molybdenum pentacarbonyl $\mathrm{Mo}(\mathrm{CO})_{5} \mathrm{~L}$ (PCO) or the molybdenum tetracarbonyl complex $\mathrm{Mo}(\mathrm{CO})_{4} \mathrm{~L}_{2}$ (TCO) $\left(\mathrm{L}=\mathrm{Ph}_{2} \mathrm{PCH}_{2} \mathrm{CH}_{2} \mathrm{SO}_{3} \mathrm{Na}\right)$. It is known that the liposome membrane allows the inclusion of a wide variety of lipophilic or amphipathic substances such as, drugs and proteins. Thus, the incorporation of a bilayer-forming metallosurfactant into a phospholipid bilayer was considered to be a rational choice. The simplest procedure to obtain mixed vesicles involves the

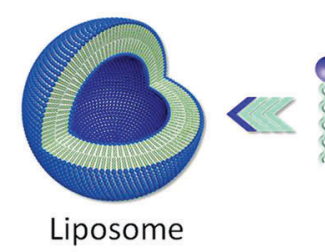

Polar head group Hydrophobic tail
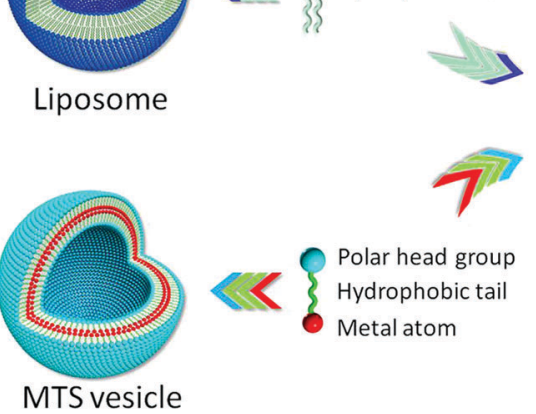

Polar head group Metal atom Hydrophobic tail

Scheme 1 Representation of vesicles obtained with a pure phospholipid or with a metallosurfactant, and when they are mixed. 

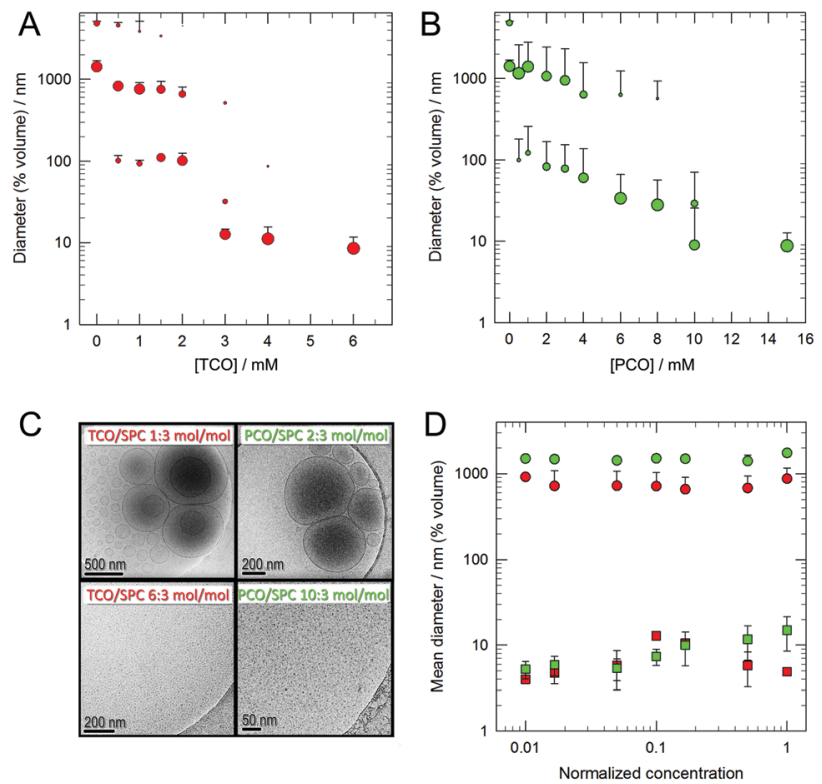

Fig. 1 (A and B) Size distribution of (A) TCO/SPC aggregates (red circles) and (B) PCO/SPC aggregates (green circles) as a function of the concentration of MTS obtained by DLS analysis $(n \geq 3)$. The molar concentration of SPC was kept constant at $3 \mathrm{mM}$. The area of the symbols is proportional to the percentage of vesicles of a given diameter. (C) Cryo-TEM images of mixed systems of MTS and SPC. (D) Size of the aggregates as a function of the relative concentration upon dilution mixed systems constituted by TCO/SPC $6: 18 \mathrm{mM} / \mathrm{mM}$ (red circles) and $6: 3 \mathrm{mM} / \mathrm{mM}$ (red squares) and PCO/SPC $6: 18 \mathrm{mM} / \mathrm{mM}$ (green circles) and $6: 1.8 \mathrm{mM} / \mathrm{mM}$ (green squares). The given concentrations correspond to the initial concentrations (normalized concentration $=1$ ).

preparation of a homogeneous dry thin film of the substances by rotary evaporation of an organic solution, subsequent addition of aqueous medium and finally vortexing. Under these conditions the system spontaneously generates the most stable aggregates formed by intimate mixing of these substances. In our case, and as determined by dynamic light scattering (DLS) analysis, a progressive increase of the MTS/SPC molar ratio produced a significant change in the size distribution of the aggregates (Fig. 1A and B). As expected, pure SPC ([MTS] = 0) forms liposomes with a diameter of $1000 \mathrm{~nm}$ or greater but, when they are prepared with MTS, the size of the aggregates decreases with increasing MTS concentration. Thus, the liposome bilayer is affected by the presence of the MTS, which is indicative of the interaction of the MTS with the liposome membrane. It should be noted that there is a break point at about 3 and $10 \mathrm{mM}$ for TCO (Fig. 1A) and PCO (Fig. 1B), respectively. Beyond these concentrations, very small aggregates are formed which, based on their size (about $10 \mathrm{~nm}$ ), are compatible with the formation of micellar or bicellar structures. In fact, the addition of Triton X-100 to the suspensions at solubilising concentrations causes the disruption of the vesicles and the formation of mixed micelles of about one order of magnitude smaller (Fig. S1, ESI $\dagger$ ). In contrast, when the surfactant is added to the small aggregates, there is only a decrease of a few nanometers in size (Fig. S1, ESI $\dagger$ ). In addition, all the former aggregates showed physical stability at room temperature and $4{ }^{\circ} \mathrm{C}$ for at least 15 days, as determined by DLS (Fig. S1, ESI $\dagger$ ).

The nature of the aggregates was also studied by other independent techniques. Cryo-TEM images reveal the presence of mostly unilamellar vesicles in the first range of the MTS/SPC ratio, and the presence of very small structures in the second (Fig. 1C). The existence of unilamellar vesicles can be explained by the incorporation of a negative substance into the liposome bilayer. $Z$-potential measurements (Table S1, ESI $\dagger$ ) show that the incorporation of MTS into the liposome increases the negative charge of the resulting aggregates. This negative charge causes intermembrane repulsions and, consequently, promotes the formation of unilamellar aggregates. The results obtained with the previous techniques are congruent and indicate that, first, despite the differences of size and shape, MTS and SPC co-aggregate in a bilayer arrangement and, second, that at a given critical ratio, these differences collapse the membrane and beyond this point only mixed small structures are formed.

The stability of both mixed vesicles and small aggregates upon dilution was checked by DLS measurements. The progressive dilution (two orders of magnitude) of concentrated samples showed no change in the size of the aggregates, either vesicles or small aggregates, formed with TCO or PCO (Fig. 1D). Moreover, this stability was maintained for, at least, 24 hours (Fig. S2, ESI $\dagger$ ). The same stability upon dilution was observed when the MTS/SPC vesicles were previously extruded using membranes with a pore size of $100 \mathrm{~nm}$ (Fig. S2C and D, ESI†). Different controls were employed. The negative ones were suspensions of SPC liposomes (extruded through 100 or $1000 \mathrm{~nm}$ poremembranes), which, as expected, showed no vesicle disaggregation. The positive control was a bicellar suspension (an initial diameter of about $20 \mathrm{~nm}$ ), which is known to disaggregate upon dilution and form micron-sized liposomes (Fig. S3, ESI $\dagger$ ). ${ }^{5}$

At this point, our results show that MTS/SPC systems allow stable aggregates that contain metal atoms in their hydrophobic domain to be obtained. The MTS to SPC molar ratio modulates the type and size of these aggregates, since vesicles (metallosomes) and small structures of about $10 \mathrm{~nm}$ can be obtained. Thus, it is possible to obtain large metallosomes of hundreds of nanometers with an aqueous compartment that, in turn, can also entrap water soluble substances. In order to obtain a homogeneous vesicle distribution with controlled size, these metallosomes can be downsized by several standard methods, as is the case of liposomes (extrusion, homogenization, etc.). ${ }^{6}$ Moreover, the presence of phospholipids opens the way for the preparation of surface modified aggregates by derivatization. ${ }^{7}$ At the same time, the sulphonate group of MTS enables these metal-containing structures to incorporate, for example, polymers with amino groups, as is the case of chitosan, since a strong interaction between both the groups has been described. ${ }^{8}$ Thus, a broad spectrum of possibilities is on the horizon of these new metal structures.

One of these opportunities is the use of MTS as carbon monoxide releasing molecules (CORMs). It has been shown that the local delivery of $\mathrm{CO}$ has important uses in medicine. ${ }^{9,10}$ In our case, the metal atom (Mo) of the MTS is linked to several 


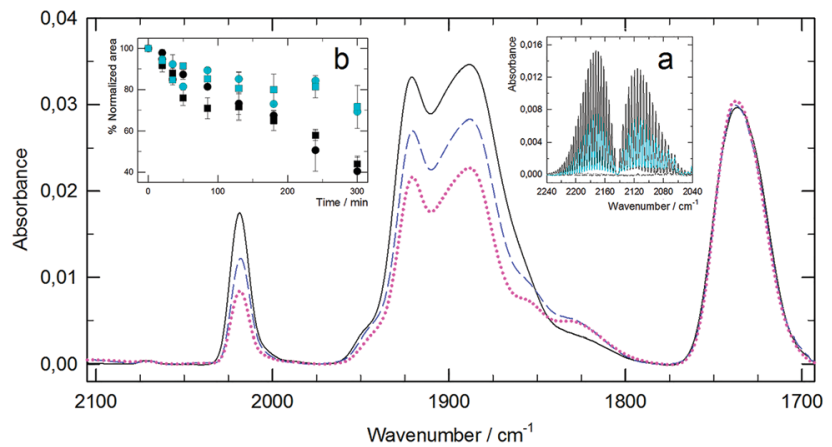

Fig. 2 Infrared spectra of TCO/SPC 1:3 mol/mol under irradiation with UVA (365 nm) at 0 (black), 85 (blue) and 300 min (magenta). Inset a: Spectra of the free space of an infrared cell partially filled with a suspension of PCO/SPC 1:3 mol/mol kept in the dark for 60 min (black dotted line), and under irradiation for 60 min with UVA (black continuous line) or visible light (cyan continuous line). Inset $b$ : Changes in the relative area of the peak at $2070 \mathrm{~cm}^{-1}$ of PCO/SPC $1: 3 \mathrm{~mol} / \mathrm{mol}$ (circles) and $10: 3 \mathrm{~mol} / \mathrm{mol}$ (squares) upon illumination with UVA (black) or visible light (cyan).

CO groups and these molecules are good candidates to release $\mathrm{CO}$ under certain conditions. Therefore, a study was carried out on the PCO and TCO metal complexes, as well as their respective metallosomes and small aggregates, as CORMs. Since there is controversy about some classical assays for the detection of $\mathrm{CO}$ release, ${ }^{10,11}$ in order to circumvent these problems we have detected the release of CO from PCO and TCO aggregates with SPC into a $\mathrm{CaF}_{2}$ infrared cell. First, as a reference, the cell was filled with $\mathrm{CO}$, and the well-known vibration-rotation spectrum of $\mathrm{CO}$ was obtained (inset a, Fig. 2). After eliminating the $\mathrm{CO}$, the required volume of the samples was placed into the cell so that the infrared beam passed through the unfilled part when maintained in a vertical position. When the sample was kept in the dark, the acquired spectra showed no presence of CO (inset a, Fig. 2). However, after illuminating the samples with visible or UVA light, the presence of $\mathrm{CO}$ in the gas phase was clearly detected. Thus, our MTS can be classified as photo-CORMs, and the release of $\mathrm{CO}$ can be modulated via light irradiation. Once the release of $\mathrm{CO}$ was confirmed, its evolution was monitored by FTIR spectroscopy using dry aliquots of samples taken during irradiation. Fig. 2 shows the spectra corresponding to TCO/SPC $1: 3 \mathrm{~mol} / \mathrm{mol}$ before and during irradiation with UVA. As can be observed, there is a noticeable change in the CO bands centered at 2019, 1921 and $1888 \mathrm{~cm}^{-1}$, as a result of the release of CO. Similar spectra with other characteristic CO bands were obtained in the case of PCO mixed systems (Fig. S4, ESI $\dagger$ ). These changes in the $\mathrm{CO}$ bands were characterized using the normalized area ratio between the MTS CO bands $\left(2071 \mathrm{~cm}^{-1}\right.$ and $2019 \mathrm{~cm}^{-1}$ for PCO and TCO, respectively) and the SPC CO band at $1730 \mathrm{~cm}^{-1}$, since this group is not altered during irradiation. Inset $b$ in Fig. 2 (PCO mixed systems) indicates that the type of molecular assembly has no influence on $\mathrm{CO}$ release. It is also noticeable that not only UVA irradiation triggers CO release, but the gas is also produced upon illumination with visible light.

Finally, two types of tests were carried out to characterize the interaction of the MTS/SPC aggregates with biological systems.

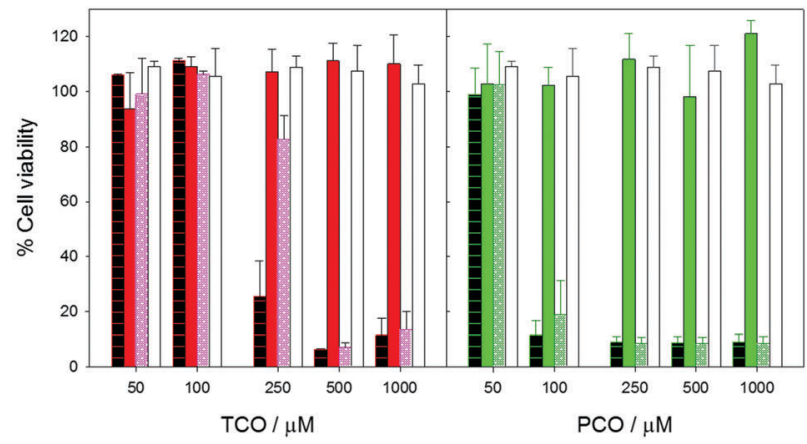

Fig. 3 Human dermal fibroblast cell viability after exposure for $24 \mathrm{~h}(n \geq 2)$, to TCO (red) or PCO (green) administered in free form (dashed) or mixed with SPC as vesicles (plain) or small aggregates (dotted). The MTS to SPC molar ratio was $1: 3$ for TCO and PCO vesicles; $6: 3$ for TCO/SPC small aggregates; and $10: 3$ for PCO/SPC small aggregates. Pure SPC liposomes (white) were used as blanks and in these incubations SPC concentrations were the same as those of the respective treatments of mixtures of SPC and MTS.

First, their 24 h-toxicity was checked by the XTT assay using human fibroblast cell cultures. The results are shown in Fig. 3, providing evidence that all the vesicular structures (metallosomes) are much less toxic than the corresponding small aggregates or the free forms of the MTS, and that PCO is more toxic than TCO when it is added as free MTS or as small aggregates.

Notably, metallosomes with TCO and PCO show no toxicity in the studied range, with their behaviour being equivalent to that of pure liposomes. The maximum MTS concentration of these systems is $1000 \mu \mathrm{M}$, that is, 10 and 20 times the maximum non-toxic concentrations, respectively, of TCO and PCO systems constituted of small aggregates.

The second study was carried out to check the ability of the mixed MTS/SPC systems to act as an efficient drug delivery system. The objective was to detect the presence of MTS in cells treated with formulations containing TCO at 250 and $1000 \mu \mathrm{M}$, and PCO at 100 and $1000 \mu \mathrm{M}$. The cells were analyzed by micro-FTIR microscopy using synchrotron radiation (ALBA synchrotron, MIRAS beamline, Spain). The characteristic CO vibration band of the MTS was detected in the cells incubated at $1 \mathrm{mM}$ of TCO or PCO (both as metallosomes or small aggregates), and a low intensity band was also observed in some cells treated with TCO metallosomes at $250 \mu \mathrm{M}$. Fig. 4 shows the results for the TCO/SPC metallosomes normalized to the total protein content of the samples (amide I band at $1650 \mathrm{~cm}^{-1}$ ), providing evidence of the presence of the MTS in the cells. Thus, it is clear that metallosomes are able to interact and to deliver their content to the cells.

In conclusion, we have demonstrated that the simple mixing of MTS and phospholipids leads to the formation of stable metallosomes. The reported new nanosystems are stable upon dilution and, although the incorporation of metal into cells has been observed by synchrotron radiation, a drastic decrease in cell toxicity is also shown in comparison with free MTS. Due to its simplicity and versatility, this new approach could be applied to the preparation of other metal based pharmaceuticals with low toxicity. 


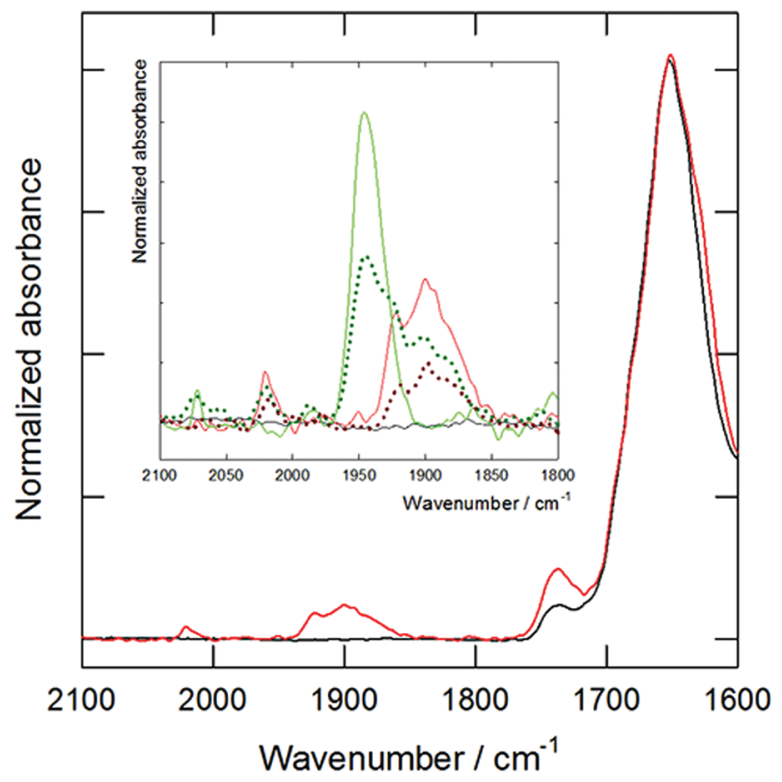

Fig. 4 Infrared spectra of fixed cell cultures of fibroblasts after incubation with a cell culture medium (black) or TCO/SPC 1000:3000 $\mu \mathrm{M} / \mu \mathrm{M}$ metallosomes (red) for $12 \mathrm{~h}$. Inset: Details of the $\mathrm{CO}$ absorption range for cultures incubated with TCO/SPC $1000: 3000 \mu \mathrm{M} / \mu \mathrm{M}$ metallosomes (red); TCO/SPC 1000:500 $\mu \mathrm{M} / \mu \mathrm{M}$ small aggregates (dotted dark red); PCO/SPC 1000:3000 $\mu \mathrm{M} / \mu \mathrm{M}$ metallosomes (green); and PCO/SPC $1000: 300 \mu \mathrm{M} / \mu \mathrm{M}$ small aggregates (dotted dark green).

This work was supported by Spanish MINECO-FEDER grants (BIO2015-67358-C2-2-P and CTQ2015-70371-REDT) and by Generalitat de Catalunya (2014SGR-423). This work is dedicated to the memory of Prof. Silvia Atrian, who was the leader of our group and prematurely passed away in the winter of 2016.

\section{Notes and references}

1 (a) P. C. Griffiths, I. A. Fallis, T. Chuenpratoom and R. Watanesk, Adv. Colloid Interface Sci., 2006, 122, 107-117; (b) T. Owen and
A. Butler, Coord. Chem. Rev., 2011, 255, 678-687; (c) R. Kaur and S. K. Mehta, Coord. Chem. Rev., 2014, 262, 37-54; (d) A. Mechler, B. D. Stringer, M. S. H. Mubin, E. H. Doeven, N. W. Phillips, J. RuddSchmidt and C. F. Hogan, Biochim. Biophys. Acta, 2014, 1838, 2939-2946; (e) L. D. Wickramasinghe, S. Mazumder, S. Gonawala, M. M. Perera, H. Baydoun, B. Thapa, L. Li, L. Xie, G. Mao, Z. Zhou, H. B. Schlegel and C. N. Verani, Angew. Chem., Int. Ed., 2014, 53(52), 14462-14467; $(f)$ Y. Chen, Q. Zhu, X. Cui, W. Tang, H. Yang, Y. Yuan and A. Hu, Chem. - Eur. J., 2014, 20(39), 12477-12482; (g) L. J. Lalgee, L. Griersona, R. A. Fairman, G. E. Jaggernauth, A. Schulte, R. Benz and M. Winterhalter, Biochim. Biophys. Acta, 2014, 1838, 1247-1254.

2 (a) E. Parera, F. Comelles, R. Barnadas and J. Suades, Chem. Commun., 2011, 47, 4460-4462; (b) E. Parera, M. Marín-García, R. Pons, F. Comelles, J. Suades and R. Barnadas-Rodríguez, Organometallics, 2016, 35, 484-493.

3 (a) Y. Perrie, F. Crofts, A. Devitt, H. R. Griffiths, E. Kastner and V. Nadella, Adv. Drug Delivery Rev., 2016, 99A, 85-96; (b) B. S. Pattni, V. V. Chupin and V. P. Torchilin, Chem. Rev., 2015, 115, 10938-10966.

4 (a) J. F. Hainfeld, F. R. Furuya and R. D. Powell, J. Struct. Biol., 1999, 127(2), 152-160; (b) K. Osada, H. Cabral, Y. Mochida, S. Lee, K. Nagata, T. Matsuura, M. Yamamoto, Y. Anraku, A. Kishimura, N. Nishiyama and K. Kataoka, J. Am. Chem. Soc., 2012, 134, 13172-13175.

5 M. Li, H. H. Morales, J. Katsaras, N. Kucerka, Y. Yang, P. M. Macdonald and M.-P. Nieh, Langmuir, 2013, 29, 15943-15957.

6 (a) A. Hinna, F. Steiniger, S. Hupfeld, P. Stein, J. Kuntsche and M. Brandl, J. Liposome Res., 2015, 26(1), 11-20; (b) R. Barnadas and M. Sabés, Methods Enzymol., 2003, 367, 28-46.

7 (a) A. S. Manjappa, K. R. Chaudhari, M. P. Venkataraju, P. Dantuluri, B. Nanda, Ch. Sidda, K. K. Sawant and R. S. Murthy, J. Controlled Release, 2011, 150, 2-22; (b) A. Accardo and G. Morelli, Biopolymers, 2015, 104(5), 462-479; (c) R. R. Sawant and V. P. Torchilin, AAPS J., 2012, 14(2), 303-315.

8 (a) Q. Yua, S. Denga and G. Yu, Water Res., 2008, 42, 3089-3097; (b) R. Barnadas-Rodríguez, Macromol. Chem. Phys., 2013, 213, 99-106.

9 (a) R. Motterlini and L. E. Otterbein, Nat. Rev. Drug Discovery, 2010, 9(9), 728-743; (b) A. C. Kautz, P. C. Kunz and C. Janiak, Dalton Trans., 2016, 45, 18045-18063; (c) U. Hasegawa, A. J. van der Vlies, E. Simeoni, Ch. Wandrey and J. A. Hubbell, J. Am. Chem. Soc., 2010, 132, 18273-18280.

10 G. J. L. Bernardes and S. García-Gallego, Angew. Chem., Int. Ed., 2014, 53, 9712-9721.

11 (a) S. McLean, B. E. Mann and R. K. Poole, Anal. Biochem., 2012, 427, 36-40; (b) S. H. Heinemann, T. Hoshi, M. Westerhausend and A. Schiller, Chem. Commun., 2014, 50, 3644-3660; (c) M. Chaves-Ferreira, I. S. Albuquerque, D. Matak-Vinkovic, A. C. Coelho, S. M. Carvalho, L. M. Saraiva, C. C. Romão and G. J. L. Bernardes, Angew. Chem., Int. Ed., 2015, 54, 1172-1175. 